\title{
Integrated Sachs-Wolfe effect: Large scale structure correlation
}

\author{
Asantha Cooray* \\ Theoretical Astrophysics, California Institute of Technology, Pasadena, California 91125
}

(Received 17 December 2001; published 30 April 2002)

\begin{abstract}
I discuss the correlation between the late-time integrated Sachs-Wolfe (ISW) effect in the cosmic microwave background $(\mathrm{CMB})$ temperature anisotropies and the large scale structure of the local universe. This correlation has been proposed and studied in the literature as a probe of the dark energy and its physical properties. I consider a variety of large scale structure tracers suitable for a detection of the ISW effect via a cross correlation. In addition to luminous sources, I suggest the use of tracers such as dark matter halos or galaxy clusters. A suitable catalogue of mass selected halos for this purpose can be constructed with upcoming wide-field lensing and Sunyaev-Zel'dovich (SZ) effect surveys. With multifrequency data, the presence of the ISW-large scale structure correlation can also be investigated through a cross-correlation of the frequency cleaned SZ and CMB maps. While convergence maps constructed from lensing surveys of the large scale structure via galaxy ellipticities are less correlated with the ISW effect, lensing potentials that deflect CMB photons are strongly correlated and allow, probably, the best mechanism to study the ISW-large scale structure correlation with $\mathrm{CMB}$ data alone.
\end{abstract}

DOI: 10.1103/PhysRevD.65.103510

PACS number(s): 98.80.Es, 95.85.Nv

\section{INTRODUCTION}

The cosmic microwave background (CMB) temperature fluctuations are now a well known probe of cosmology [1]. In addition to the dominant anisotropy contribution at the last scattering surface [2], CMB photons, while transiting to us, also encounter the large scale structure. Consequently, modifications are imprinted on the temperature with a regeneration of a new set of secondary anisotropies. In general, large scale structure affects CMB through two distinct processes: gravity and Compton scattering. The modifications due to gravity arise from frequency changes via gravitational redshifts and blueshifts $[3,4]$, through deflections involving gravitational lensing [5,6], and time delays [7]. During the reionized epoch, photons can both generate and erase primary fluctuations through scattering via free electrons [8,9].

Here, I discuss an effect due to the gravitational redshift commonly known in the literature as the integrated SachsWolfe (ISW [3]) effect at late times. The temperature fluctuations in the ISW effect result from the differential gravitational redshift from photons climbing in and out of time evolving potential perturbations from last scattering surface to the present day. In currently popular cold dark matter cosmologies with a cosmological constant, significant contributions arise at redshifts of cosmological constant domination $(z \lesssim 2)$, at, on, and above the scale of the horizon at the time of decay. When projected on the sky, the ISW effect contributes at large angular scales and has a power spectrum that scales with the wave number as $k^{-5}$ times the linear density field power spectrum [10]. This is in contrast to most other contributions to $\mathrm{CMB}$ temperature fluctuations from the local universe, such as the well known thermal SunyaevZel'dovich (SZ [8]) effect, that peak at small angular scales and scale with the wave number as $k^{-1}$.

Since time evolving potentials that contribute to the ISW

*Email address: asante@caltech.edu effect may also be probed by observations of the large scale structure, it is then expected that the ISW effect may be correlated with certain tracers. The presence of the ISW effect can then be detected via a cross-correlation of the CMB temperature fluctuations at large angular scales and the fluctuations in an appropriate tracer field. Since the ISW contribution is sensitive to how one models cosmology at late times, such as the presence of a dark energy component and its physical properties, for example, the ratio of dark energy pressure to density, the correlation between the CMB temperature and tracer fields has been widely discussed in the literature [11].

Though several attempts have already been made to crosscorrelate the ISW effect, using the best Cosmic Background Explorer (COBE) temperature map, and foreground sources, such as x-ray and radio galaxies, there is, so far, no clear detection of the correlation signal [12]. As we discuss later, these nondetections are not surprising given the large sample variance associated with the correlating part of the temperature fluctuations, i.e., the ISW effect, with contribution to the variance coming from primary temperature fluctuations. Even for the best case scenario involving a whole-sky observation and no noise contribution to the tracer field, the expected cumulative signal-to-noise ratio for the ISW-large scale structure correlation is at most a 10. If the ISW-large scale structure correlation is to be used as a probe of cosmological and astrophysical properties, it is certainly necessary to study in detail what tracers are best correlated with the ISW effect and why.

In this paper I address this question by studying in detail the correlation between the ISW effect and the large scale structure. Since I am primarily interested in understanding what types of tracers are best suited to detect the correlation, I will consider a variety of large scale structure observations and tracers. These include luminous sources, such as galaxies or active galactic nuclei (AGN) at different wavelengths, the dark matter halos, or galaxy clusters, that describe the large scale clustering of the universe, gravitational lensing, and 
other contributions to the CMB from the large scale structure, such as the thermal SZ effect. Unlike previous studies $[13,14]$, I do not consider applications of the crosscorrelation involving the determination of cosmological parameters and dark energy properties.

In Sec. II I outline the correlation between the ISW effect and the large scale structure. In Sec. III I discuss my results and study which tracers are best suited for correlation studies. I suggest that in addition to tracers involving sources such as galaxies or AGNs, dark matter halos that contain these sources may also be suitable for a detection of the ISW effect via a cross-correlation. The best probe of the ISWlarge scale structure correlation is the lensing effect on CMB photons. Since one can extract information related to lensing deflections from a quadratic statistic on temperature data $[15,16]$, this allows one to use all-sky CMB anisotropy maps alone, such as those expected from the Planck surveyor, to extract the ISW-large scale structure correlation.

\section{ISW-LARGE SCALE STRUCTURE CORRELATION}

The integrated Sachs-Wolfe effect [3] results from the late time decay of gravitational potential fluctuations. The resulting temperature fluctuations in the $\mathrm{CMB}$ can be written as

$$
T^{\mathrm{ISW}}(\hat{\mathbf{n}})=-2 \int_{0}^{r_{0}} d r \dot{\Phi}(r, \hat{\mathbf{n}} r),
$$

where the overdot represents the derivative with respect to conformal distance, or equivalently look-back time, from the observer at redshift $z=0$

$$
r(z)=\int_{0}^{z} \frac{d z^{\prime}}{H\left(z^{\prime}\right)} .
$$

Here, the expansion rate for adiabatic cold dark matter (CDM) cosmological models with a cosmological constant is

$$
H^{2}=H_{0}^{2}\left[\Omega_{m}(1+z)^{3}+\Omega_{K}(1+z)^{2}+\Omega_{\Lambda}\right],
$$

where $H_{0}$ can be written as the inverse Hubble distance today $H_{0}^{-1}=2997.9 h^{-1}$ Mpc. I follow the conventions that in units of the critical density $3 H_{0}^{2} / 8 \pi G$, the contribution of each component is denoted $\Omega_{i}, i=c$ for the CDM, $g$ for the baryons, and $\Lambda$ for the cosmological constant. We also define the auxiliary quantities $\Omega_{m}=\Omega_{c}+\Omega_{b}$ and $\Omega_{K}=1-\Sigma_{i} \Omega_{i}$, which represent the matter density and the contribution of spatial curvature to the expansion rate, respectively.

Writing multipole moments of the temperature fluctuation field $T(\hat{\mathbf{n}})$,

$$
a_{l m}=\int d \hat{\mathbf{n}} T(\hat{\mathbf{n}}) Y_{l}^{m *}(\hat{\mathbf{n}}),
$$

I can formulate the angular power spectrum as

$$
\left\langle a_{l_{1} m_{1}}^{*} a_{l_{2} m_{2}}\right\rangle=\delta_{l_{1} l_{2}}^{\mathrm{D}} \delta_{m_{1} m_{2}}^{\mathrm{D}} C_{l_{1}} .
$$

For the ISW effect, multipole moments are

$$
a_{l m}^{\mathrm{ISW}}=i^{l} \int \frac{d^{3} \mathbf{k}}{2 \pi^{2}} \int d r \dot{\Phi}(\mathbf{k}) I_{l}(k) Y_{l}^{m}(\hat{\mathbf{k}})
$$

with $I_{l}^{\mathrm{ISW}}(k)=\int d r W^{\mathrm{ISW}}(k, r) j_{l}(k r)$, and $W^{\mathrm{ISW}}$ is the window function for the ISW effect, Here, I have used the Rayleigh expansion of a plane wave

$$
e^{i \mathbf{k} \cdot \hat{\mathbf{n}} r}=4 \pi \sum_{l m} i^{l} j_{l}(k r) Y_{l}^{m *}(\hat{\mathbf{k}}) Y_{l}^{m}(\hat{\mathbf{n}}) .
$$

In order to calculate the power spectrum involving the time-derivative of potential fluctuations, I make use of the cosmological Poisson equation [17]. In Fourier space, I can relate fluctuations in the potential to the density field as

$$
\Phi=\frac{3}{2} \frac{\Omega_{m}}{a}\left(\frac{H_{0}}{k}\right)^{2}\left(1+3 \frac{H_{0}^{2}}{k^{2}} \Omega_{K}\right)^{-2} \delta(k, r) .
$$

Thus the derivative of the potential can be related to a derivative of the density field and the scale factor $a$. In linear theory, the density field may be scaled backwards to a higher redshift by the use of the growth function $G(z)$, where $\delta(k, r)=G(r) \delta(k, 0)[18]$

$$
G(r) \propto \frac{H(r)}{H_{0}} \int_{z(r)}^{\infty} d z^{\prime}\left(1+z^{\prime}\right)\left(\frac{H_{0}}{H\left(z^{\prime}\right)}\right)^{3} .
$$

Note that in the matter dominated epoch $G \propto a=(1+z)^{-1}$. It is, therefore, convenient to define a new variable $F(r)$ such that $F \equiv G / a$.

Since I will be discussing the correlation between the ISW effect and the tracers of the large scale structure density field, for simplicity, I can write the power spectrum of the ISW effect in terms of the density field such that

$$
C_{l}^{\mathrm{ISW}}=\frac{2}{\pi} \int k^{2} d k P_{\delta \delta}(k)\left[I_{l}^{\mathrm{ISW}}(k)\right]^{2},
$$

where

$$
I_{l}^{\mathrm{ISW}}(k)=\int_{0}^{r_{0}} d r W^{\mathrm{ISW}}(k, r) j_{l}(k r),
$$

with

$$
W^{\mathrm{ISW}}(k, r)=-3 \Omega\left(\frac{H_{0}}{k}\right)^{2} \dot{F} .
$$

Here I have introduced the power spectrum of density fluctuations

$$
\left\langle\delta(\mathbf{k}) \delta\left(\mathbf{k}^{\prime}\right)\right\rangle=(2 \pi)^{3} \delta^{\mathrm{D}}\left(\mathbf{k}+\mathbf{k}^{\prime}\right) P(k),
$$

where

$$
\frac{k^{3} P_{\delta \delta}(k)}{2 \pi^{2}}=\delta_{H}^{2}\left(\frac{k}{H_{0}}\right)^{n+3} T^{2}(k)
$$


in linear perturbation theory. Here $\delta_{H}$ is the amplitude of the present-day density fluctuations at the Hubble scale and I use the fitting formulas of [19] in evaluating the transfer function $T(k)$ for CDM models.

The cross-correlation between the ISW effect and another tracer of the large scale structure can be similarly constructed by taking the multipolar expansion of the tracer field. Following arguments similar to the above, we obtain this crosscorrelation as

$$
C_{l}^{\mathrm{ISW}-\mathrm{X}}=\frac{2}{\pi} \int k^{2} d k P_{\delta \delta}(k) I_{l}^{\mathrm{ISW}}(k) I_{l}^{\mathrm{X}}(k),
$$

where

$$
I_{l}^{\mathrm{X}}(k)=\int_{0}^{r_{0}} d r W^{\mathrm{X}}(k, r) j_{l}(k r),
$$

with the window function for the $\mathrm{X}$-field as $W^{\mathrm{X}}(k, r)$. To estimate how well the tracer field correlates with the ISW effect, I will introduce the correlation coefficient $r_{\text {ISW-X }}$ such that

$$
r_{\mathrm{ISW}-\mathrm{X}}=\frac{C_{l}^{\mathrm{ISW}-\mathrm{X}}}{\sqrt{C_{l}^{\mathrm{ISW}} C_{l}^{\mathrm{X}}}},
$$

where the power spectrum of the X-field follows from above

$$
C_{l}^{\mathrm{X}}=\frac{2}{\pi} \int k^{2} d k P_{\delta \delta}(k)\left[I_{l}^{\mathrm{X}}(k)\right]^{2} .
$$

A correlation coefficient of $\sim 1$ suggests that the tracer field is well correlated with the ISW effect. One of the goals here is to investigate which tracer fields lead to correlation coefficients close to 1 .

Using the covariance of the cross-correlation power spectrum, we can write the signal-to-noise ratio for the detection of the ISW-large scale structure as

$$
\begin{aligned}
\left(\frac{S}{N}\right)^{2}= & f_{\text {sky }} \sum_{l=l_{\min }}^{l_{\max }}(2 l+1) \\
& \times \frac{\left[C_{l}^{\mathrm{ISW}-\mathrm{X}}\right]^{2}}{\left[C_{l}^{\mathrm{ISW}-\mathrm{X}}\right]^{2}+\left(C_{l}^{\mathrm{ISW}}+C_{l}^{\mathrm{N}_{\mathrm{ISW}}}\right)\left(C_{l}^{\mathrm{X}}+C_{l}^{\mathrm{N}_{\mathrm{X}}}\right)},
\end{aligned}
$$

where noise contributions to the ISW map and the tracer map are given by $C_{l}^{\mathrm{N}_{\mathrm{ISW}}}$ and $C_{l}^{\mathrm{N}_{\mathrm{X}}}$, respectively.

In the case of the ISW effect, the noise contributions are effectively

$$
C_{l}^{\mathrm{N}_{\mathrm{ISW}}}=C_{l}^{\mathrm{CMB}}+C_{l}^{\mathrm{det}},
$$

where $C_{l}^{\mathrm{CMB}}$ is the total temperature fluctuation contribution, including the ISW contribution, while $C_{l}^{\text {det }}$ is any detector noise contribution. As we find later, it is the cosmic variance associated with $C_{l}^{\mathrm{CMB}}$, and not the detector noise, that limits the signal-to-noise ratio for the detection of the ISW-large scale structure correlation. Therefore most of the results I present here apply equally well to both Microwave Anisotropy Probe (MAP) and Planck surveyor missions as they are cosmic variance limited at large scales. I will also discuss tracer fields, such as the SZ effect, involving information extracted using temperature data from these satellites. In such cases, when the signal-to-noise is different, I will separately quote them for MAP and Planck.

In Eq. (19), I have approximated the number of independent modes available for the measurement of the crosscorrelation as $f_{\text {sky }}(2 l+1)$. The summation generally starts from $l_{\min }=2$, though, when observations are limited to a fraction of the sky, low multipoles are not independent and there is a substantial reduction in the number of independent modes. To account for the loss of low multipole modes, one can approximate the summation such that $l_{\min } \sim \pi / \theta_{\text {sky }}$ where $\theta_{\text {sky }}$ is the survey size in the smallest dimension. Since the ISW effect peaks at large angular scales, where in some cases the cross-correlation also peaks, it is crucial that the reduction in the signal-to-noise ratio due to the partial sky coverage be considered.

Since I will be primarily interested in all-sky temperature maps from upcoming satellite missions, these issues are of minor concern. I will assume a usable fraction of $f_{\text {sky }}$ $=0.65$ for such missions due to sky cuts involving the galactic plane. This still leads to almost independent modes at each multipole. When cross-correlating with surveys of limited area tracer fields, however, the detectability of the ISW effect via the attempted correlation may crucially depend on the sky coverage of the tracer field.

Note that an expression of the type in Eq. (10) can be evaluated efficiently with the Limber approximation [20]. Here I employ a version based on the completeness relation of spherical Bessel functions

$$
\left.\int d k k^{2} F(k) j_{l}(k r) j_{l}\left(k r^{\prime}\right) \approx \frac{\pi}{2} d_{A}^{-2} \delta^{\mathrm{D}}\left(r-r^{\prime}\right) F(k)\right|_{k=\frac{l}{d_{A}}},
$$

where the assumption is that $F(k)$ is a slowly varying function. Using this, I obtain a useful approximation for the power spectrum for sufficiently high $l$ values, $l \sim$ few hundred, as

$$
C_{l}^{\mathrm{ISW}}=\int d r \frac{\left[W^{\mathrm{ISW}}\right]^{2}}{d_{A}^{2}} P_{\dot{\Phi} \dot{\Phi}}\left[k=\frac{l}{d_{A}}, r\right] .
$$

Here, the comoving angular diameter distance, in terms of the radial distance, is

$$
d_{A}=H_{0}^{-1} \Omega_{K}^{-1 / 2} \sinh \left(H_{0} \Omega_{K}^{1 / 2} r\right) .
$$

Note that as $\Omega_{K} \rightarrow 0, d_{A} \rightarrow r$ and I define $r(z=\infty)=r_{0}$.

Although I maintained generality in all derivations, I now illustrate my results with the currently favored $\Lambda \mathrm{CDM}$ cosmological model. The parameters for this model are $\Omega_{c}$ $=0.30, \Omega_{b}=0.05, \Omega_{\Lambda}=0.65$, and $h=0.65$. With $n=1$, I adopt the COBE normalization for $\delta_{H}$ [21] of $4.2 \times 10^{-5}$, 
such that mass fluctuations on the $8 h \mathrm{Mpc}^{-1}$ scale are $\sigma_{8}$ $=0.86$, consistent with observations on the abundance of galaxy clusters [22].

\section{RESULTS}

I now discuss a variety of large scale structure tracers that can potentially be used to cross-correlate with CMB temperature data.

\section{A. ISW-source correlation}

Probably the most obvious tracer of the large scale structure density field in the linear regime is luminous sources such as galaxies at optical wavelengths and AGNs at X-rays and/or radio wavelengths. I can write the associated window function in these cases as

$$
W^{\mathrm{X}}(k, r)=b_{\mathrm{X}}(k, r) n_{\mathrm{X}}(r) G(r),
$$

where $b_{X}(k, r)$ is the scale-dependent source bias as a function of the radial distance and $n_{X}(r)$ is the normalized redshift distribution of sources such that $\int_{0}^{r_{0}} d r n_{X}(r)=1$. I will model the redshift distribution in current and upcoming source catalogues with an analytic form:

$$
n_{\mathrm{X}}(z)=\left(\frac{z}{z_{0}}\right)^{\alpha} \exp -\left(\frac{z}{z_{0}}\right)^{\beta}
$$

where $\alpha$ and $\beta$ denote the slope of the distribution at low and high $z$ 's, respectively, with a "mean" given by $\sim z_{0}$. For the purpose of this calculation I take $\alpha=\beta=1$ and vary $z_{0}$ from 0.1 to 1.5 so as to mimic the expected distribution of sources from current and upcoming catalogues as well as to cover the redshift range in which ISW contributions are generally expected.

Since any redshift dependence on bias can be included as a variation to the source redshift distribution, the scale dependence on bias is less important and I consider a scale independent bias description when illustrating our results. In fact, since the ISW effect is primarily associated with scales which are large and linear, the source bias can be well approximated with a scale independent number. Such an assumption is also fully consistent with results from numerical simulations [23], results from redshift surveys [24], and from semianalytic calculations involving the so-called halo model [25]. We take the source bias to be in the range of $1-3$ as expected for galaxies and highly biased sources such as X-ray objects. Since $C_{l}^{\mathrm{ISW}-\mathrm{X}} \propto b$, and $C_{l}^{\mathrm{X}} \propto b^{2}$, note that the correlation coefficient is independent of bias and other factors in the normalization. The bias becomes important only for estimating the signal-to-noise ratio.

In order to estimate signal-to-noise ratio, I describe the noise contribution associated with source catalogues by the finite number of sources one can effectively use to crosscorrelate with the temperature data. I can write this shotnoise contribution as

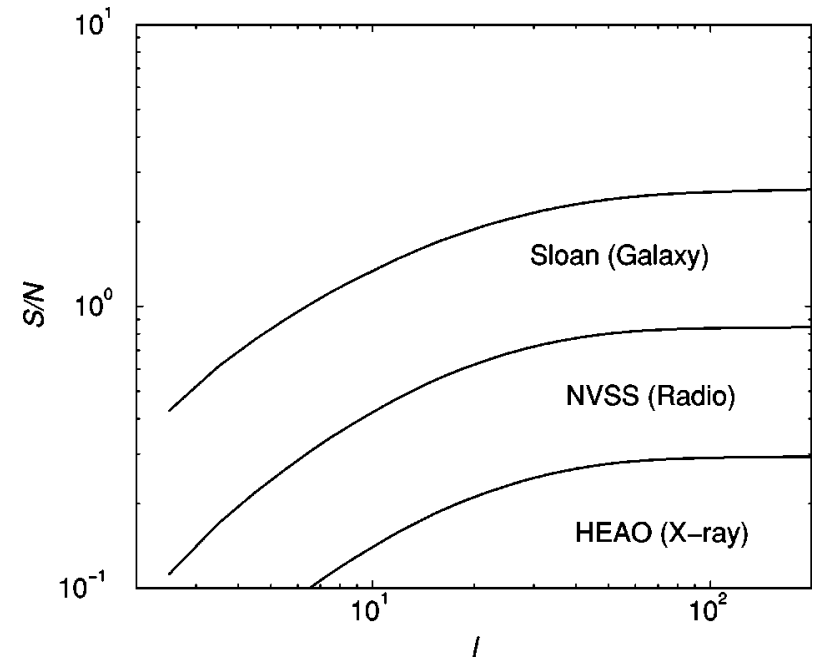

FIG. 1. The signal-to-noise ratio for the detection of source-ISW cross-correlation with a variety of sources that trace the large scale structure. The considered surveys include: HEAO x-ray catalogue, radio sources from the NVSS, and the upcoming Sloan galaxy catalogue. The signal-to-noise ratio includes the fraction of sky covered in each of these surveys. The correlation with Sloan is limited by the sample variance associated with the ISW signal while the other two catalogues have significant shot-noise associated with the limited number counts.

$$
C_{l}^{\mathrm{N}_{\mathrm{X}}}=\frac{1}{\bar{N}},
$$

where $\bar{N}$ is the surface density of sources per steradian.

In Fig. 1 I show the cumulative signal-to-noise ratio for a cross-correlation of the temperature data with the large scale structure. I have considered three source catalogues here involving the HEAO X-ray catalogue $\left(f_{\text {sky }}=1 / 3, \quad \bar{N}\right.$ $\sim 10^{3} \mathrm{sr}^{-1}$, and $\left.b_{\mathrm{X}}=3\right)$, NVSS radio sources $\left(f_{\text {sky }}=0.82\right.$, $\bar{N} \sim 2 \times 10^{5} \mathrm{sr}^{-1}$, and $b_{X}=1.6$ ) [26], and galaxy counts down to $R$ band magnitude of 25 from the Sloan Digital Sky survey $\left(f_{\text {sky }}=0.25,7 \times 10^{8} \mathrm{sr}^{-1}\right.$, and $\left.b_{X}=1\right)$ [27]. The first two catalogues have already been used for cross-correlation studies with the best COBE data [12]. The nondetection of the cross-correlation is not surprising given that I find cumulative signal-to-noise ratio values less than 1 ; this estimate will decrease once the noise contribution associated with the COBE temperature data is also included. In both cases, the shot-noise associated with the tracer field is significant. For Sloan, the limiting factor in the signal-to-noise ratio is the dominant noise contribution associated with the ISW contribution due to the sample variance associated with primary temperature fluctuations. My estimates for the signal-tonoise ratio for the ISW-Sloan correlation is consistent with previous estimates [13], where it was concluded that the detection may be challenging given the small signal-to-noise ratio values.

In addition to luminous sources, one can also crosscorrelate the temperature map with a catalogue of galaxy clusters or dark matter halos. It is expected that wide-field galaxy lensing surveys and small-angular resolution SZ sur- 
veys will allow mass selected catalogues of clusters [28]. Similar catalogues of clusters can also be compiled through wide-field galaxy surveys such as the Sloan and X-ray imaging data, such as from the proposed Dark Universe Exploration Telescope (DUET).

The redshift distribution of halos in such catalogues follows simply from analytical or numerical arguments, such as through the mass function, $d n(M, z) / d M$, calculated following Press-Schechter (PS; [29]) theory or numerical measurements [30]. Additionally, the halo bias is also well known through analytical methods [31]:

$$
b_{h}(M, z)=1+\frac{\left[\nu^{2}(M, z)-1\right]}{\delta_{c}},
$$

where $\nu(M, z)=\delta_{c} / \sigma(M, z)$ is the peak-height threshold, $\sigma(M, z)$ is the rms fluctuation within a top-hat filter at the virial radius corresponding to mass $M$, and $\delta_{c}$ is the threshold overdensity of spherical collapse. Useful fitting functions and additional information on these quantities could be found in [32]. For the purpose of this calculation I use $b_{X}$ $=\left\langle b_{M}\right\rangle$, such that the mass averaged halo bias, as a function of redshift, is

$$
\left\langle b_{M}\right\rangle(z)=\frac{1}{\bar{n}_{h}(z)} \int_{M_{\min }}^{\infty} d M \frac{d n(M, z)}{d M} b_{h}(M, z) .
$$

Here, the mean number density of halos, as a function of redshift, is given by $\bar{n}_{h}(z)=\int d M d n(M, z) / d M$.

The redshift distribution of halos follows similarly from the PS theory:

$$
n_{\mathrm{X}}(z)=\frac{1}{\bar{N}} \frac{d^{2} V}{d z d \Omega}\left[\int_{M_{\min }}^{\infty} d M \frac{d n(M, z)}{d M}\right]
$$

where

$$
\bar{N}=\int d z \frac{d^{2} V}{d z d \Omega}\left[\int_{M_{\min }}^{\infty} d M \frac{d n(M, z)}{d M}\right],
$$

with the comoving volume element given by $d^{2} V / d z d \Omega$. Note that the shot-noise associated with the halo-halo power spectrum is given by the surface density of halos $C_{l}^{\mathrm{N}_{\mathrm{X}}}$ $\equiv 1 / \bar{N}$. Even though the surface density of halos is significantly smaller than that of galaxies or sources, one gains an equivalent factor with the increase in halo bias relative to that for galaxies. Therefore, on average, I expect catalogues based on halos to produce the same order of magnitude result for signal-to-noise ratios as those based on catalogues of galaxies.

In Fig. 2 I show the cumulative signal-to-noise ratio: for detecting the ISW-halo correlation. Here, I assume all-sky catalogues of clusters down to a minimum mass limit of $10^{13}, 10^{14}$, and $10^{15} \mathrm{M}_{\odot}$. The lower limit of $10^{14} \mathrm{M}_{\odot}$ is consistent with the expected mass threshold for SZ clusters that will be detected with the planned South Pole $8 \mathrm{~m}$ Telescope outfitted with a large bolometer array [28]. This mass limit is constant over a wide range in redshift, while mass

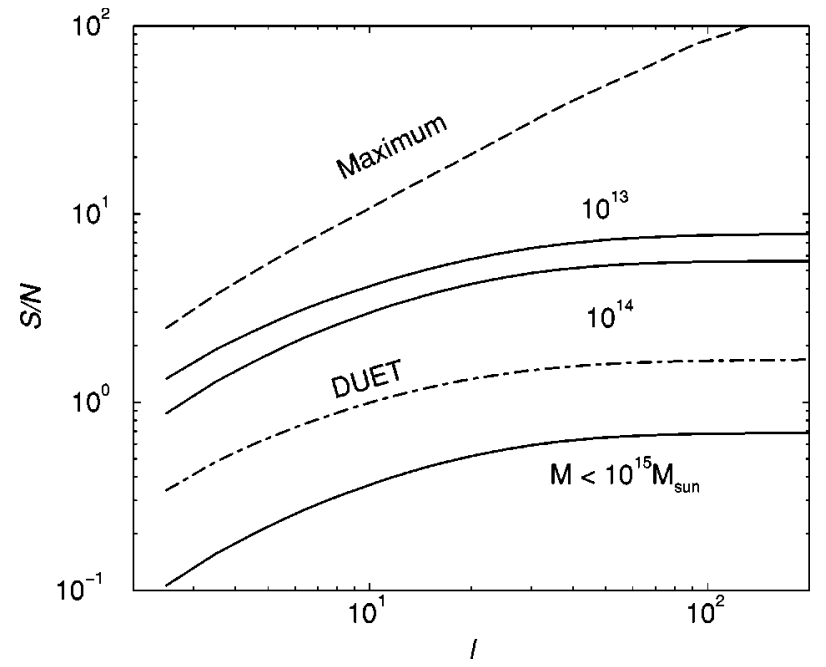

FIG. 2. The signal-to-noise ratio for the detection of source-ISW cross-correlation with dark matter halos that trace the large scale structure with low mass limits down to $10^{15}, 10^{14}$, and $10^{13} \mathrm{M}_{\odot}$ (solid lines). The long-dashed line is the maximum signal-to-noise ratio when the ISW effect can be perfectly separated from the dominant CMB data. The dot-dashed line shows an estimate for the correlation using a catalogue of clusters based on the proposed Dark Universe Exploration Telescope (DUET).

catalogues based on lensing surveys contain a redshift dependent minimum mass limit due to the redshift dependence on the lensing window function.

The decrease in the signal-to-noise ratio with the increase in mass is due to the decrease in the surface density of halos and thus the increase in the shot-noise associated with the halo side of the correlation. Though the halo surface density decreases as a function of mass, this is partly accounted for by the increase in the halo bias such that even with a low surface density of halos, one can attempt a correlation of the temperature with cluster catalogues. As an example of a temperature-source catalogue correlation that can be expected with the MAP data, I also show an estimate for the signal-to-noise ratio for a catalogue of $\sim 18000$ clusters that is expected to be compiled from the wide-field $\mathrm{x}$-ray survey by the proposed DUET mission (dot-dashed line). Though the cumulative signal-to-noise ratio is below 2, the DUET catalogue is significantly preferred over the Sloan galaxy survey due to the fact that cluster bias associated with DUET tracers can be a priori known through mass estimates based on the electron temperature data while galaxy bias may be more complicated.

\section{B. ISW-SZ correlation}

Following the derivation of the ISW-large scale structure correlation, I can also consider the cross-correlation between the ISW effect and the Sunyave-Zel'dovich thermal effect [8] due to inverse-Compton scattering of CMB photons via hot electrons. The temperature decrement due to the SZ effect can be written as the integral of pressure along the line of sight 


$$
y \equiv \frac{\Delta T}{T_{\mathrm{CMB}}}=g(x) \int d r a(r) \frac{k_{B} \sigma_{T}}{m_{e} c^{2}} n_{e}(r) T_{e}(r),
$$

where $\sigma_{T}$ is the Thomson cross section, $n_{e}$ is the electron number density, $r$ is the comoving distance, and $g(x)$ $=x \operatorname{coth}(x / 2)-4$ with $x=h \nu / k_{B} T_{\mathrm{CMB}}$ the spectral shape of the SZ effect. At the Rayleigh-Jeans (RJ) part of the CMB, $g(x)=-2$. For the rest of this paper I assume observations in the Rayleigh-Jeans regime of the spectrum. Due to the spectral dependence of the SZ effect when compared to CMB thermal fluctuations, the SZ signal can be extracted from CMB fluctuations in multifrequency data [34]. Here I use expected results from such a frequency separation with Planck and MAP data, and consider the ISW-SZ crosscorrelation by correlating the CMB and SZ maps. When calculating the signal-to-noise ratio, I will use the noise power spectra calculated in [34] for the Planck SZ and CMB maps.

Following my discussion on the ISW-large scale structure correlation, I can write the relevant window function for the SZ effect as

$$
W^{\mathrm{SZ}}(r)=g(x) \frac{k_{B} \sigma_{T} b_{g}(r) \bar{n}_{e}}{a(r)^{2} m_{e} c^{2}},
$$

where, at linear scales corresponding to the ISW effect, the pressure bias, $b_{g}$, relative to the density field follows from arguments based on the halo approach to large scale pressure fluctuations $[25,33]$ :

$$
b_{g}(z)=\int d M \frac{M}{\bar{\rho}} \frac{d n(M, z)}{d M} b_{\text {halo }}(M, z) T_{e}(M, z) .
$$

Here $T_{e}(M, z)$ is the electron temperature, which can be calculated through the virial theorem. In Eq. (32), $\bar{n}_{e}$ is the mean density of electrons today.

In Fig. 3 I show the cross-correlation between the ISW and the SZ effects. For comparison, I also show the ISW and SZ power spectra. The correlation coefficient for the ISW-SZ effect ranges from 0.3 , at $l \sim$ a few tens to 0.1 at $l \sim$ a few hundred suggesting that the ISW and the SZ contributions are not strongly correlated. This could be understood based on the fact that contributions to the SZ effect primarily come from the so-called 1-halo term of the halo model of nonlinear clustering and not the 2-halo term that traces the large scale correlations and, thus, the linear density fluctuations responsible for the ISW effect.

In the same figure I also show the signal-to-noise ratio for the detection of the ISW-SZ cross-correlation. For an experiment like Planck, I find that the signal-to-noise ratio is at the level of $\sim 4$, while for MAP, it is at the level of 0.3; this is understandable as MAP has no high frequency information for a reliable separation of the SZ effect. In order to investigate what limits the signal-to-noise ratio for the detection of the ISW-SZ correlation with a mission like Planck, I decided to set the noise contribution to the ISW effect as simply due to the ISW effect itself, instead of the total CMB power spectrum. This led to the dot-dashed line. Further removing
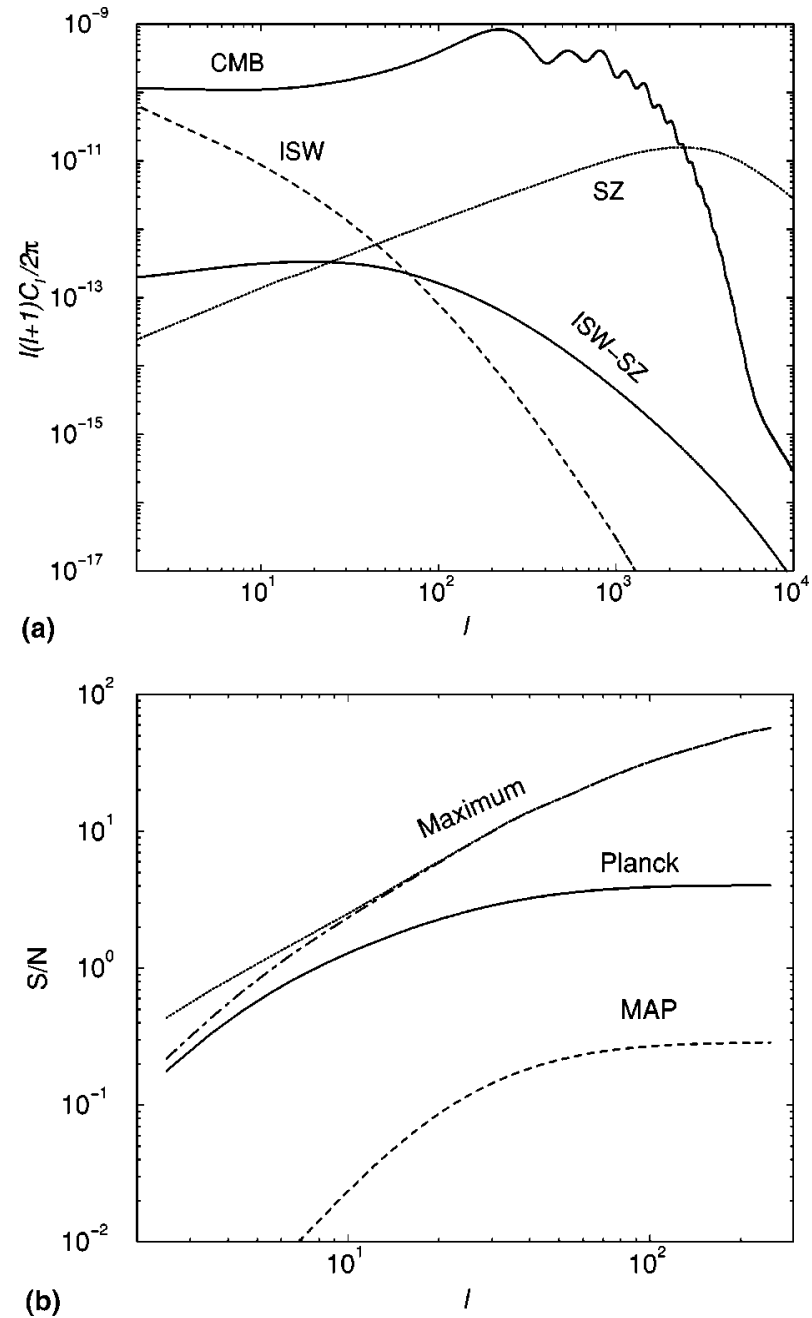

FIG. 3. Left: The cross-correlation power spectrum between the ISW and SZ effects. For comparison I also show the power spectra of ISW and SZ effects and the CMB anisotropies. Right: The cumulative signal-to-noise ratio for the detection of the ISW-SZ correlation with Planck and MAP data and using spectral dependence of the SZ contribution to separate it out from thermal CMB fluctuations. The maximum signal-to-noise ratio is when the ISW effect is separated from the dominant primary fluctuations at the last scattering surface.

the noise contribution to the SZ map, such that a perfect separation of the SZ effect is possible led to the dotted line, which converges to the previous dot-dashed line by $l \sim 10$. It is clear that the dominant noise contribution comes from the noise associated with the ISW effect, and effectively, the dominant $\mathrm{CMB}$ anisotropies at the last scattering which cannot be easily separated from the ISW contribution. If there is a useful separation scheme to extract the ISW contribution, the expected signal-to-noise ratio for the ISW-SZ correlation is at the level of $\sim 60$ suggesting a clear detection of the correlation. Though such a detection will certainly be useful for cosmological and astrophysical purposes, there is no reliable separation technique to extract the temperature fluctuations due to the ISW effect from other thermal fluctuations. 


\section{ISW-lensing correlation}

I can consider two forms of lensing: the potentials that deflect CMB photons and potentials that shear background galaxy images. The former can be constructed using quadratic statistics in the temperature data or using Fourier space statistics that are optimized to extract the lensing signal. The latter is probed in weak lensing surveys using galaxy shapes.

The deflection angle of CMB photons on the sky, $\alpha(\hat{\mathbf{n}})$ $=\nabla \phi(\hat{\mathbf{n}})$, is given by the gradient of the projected potential $\Phi$ (see, e.g., [35]),

$$
\phi(\hat{\mathbf{m}})=-2 \int_{0}^{r_{0}} d r \frac{d_{A}\left(r_{0}-r\right)}{d_{A}(r) d_{A}\left(r_{0}\right)} \Phi(r, \hat{\mathbf{m}} r) .
$$

The lensing potential can be related to the well known convergence generally encountered in conventional lensing studies involving the galaxy shear

$$
\begin{aligned}
\kappa(\hat{\mathbf{m}}) & =\frac{1}{2} \nabla^{2} \phi(\hat{\mathbf{m}}) \\
& =-\int_{0}^{r_{0}} d r \frac{d_{A}(r) d_{A}\left(r_{0}-r\right)}{d_{A}\left(r_{0}\right)} \nabla_{\perp}^{2} \Phi(r, \hat{\mathbf{m}} r),
\end{aligned}
$$

where I note that the 2D Laplacian operating on $\Phi$ is a spatial and not an angular Laplacian. Expanding the lensing potential to Fourier moments,

$$
\phi(\hat{\mathbf{n}})=\int \frac{d^{2} \mathbf{l}}{(2 \pi)^{2}} \phi(\mathbf{l}) \mathrm{e}^{i \mathbf{l} \cdot \hat{\mathbf{n}}}
$$

I can write the usually familiar quantities of convergence and shear components of weak lensing as [6]

$$
\begin{gathered}
\kappa(\hat{\mathbf{n}})=-\frac{1}{2} \int \frac{d^{2} \mathbf{l}}{(2 \pi)^{2}} l^{2} \phi(\mathbf{l}) \mathrm{e}^{i \mathbf{l} \cdot \hat{\mathbf{n}}} \\
\gamma_{1}(\hat{\mathbf{n}}) \pm i \gamma_{2}(\hat{\mathbf{n}})=-\frac{1}{2} \int \frac{d^{2} \mathbf{l}}{(2 \pi)^{2}} l^{2} \phi(\mathbf{l}) \mathrm{e}^{ \pm i 2\left(\phi_{l}-\phi\right)} \mathrm{e}^{i \mathbf{l} \cdot \hat{\mathbf{n}}} .
\end{gathered}
$$

Though these two lensing terms $\kappa$ and $\phi$ contain differences with respect to radial and wave-number weights, these differences cancel with the Limber approximation [20]. In particular, their spherical harmonic moments are simply proportional

$$
\begin{aligned}
\phi_{l m} & =-\frac{2}{l(l+1)} \kappa_{l m}=\int d \hat{\mathbf{n}} Y_{l}^{m *}(\hat{\mathbf{n}}) \phi(\hat{\mathbf{n}}) \\
& =i^{l} \int \frac{d^{3} \mathbf{k}}{2 \pi^{2}} \delta(\mathbf{k}) Y_{l}^{m} *(\hat{\mathbf{k}}) I_{l}^{\mathrm{len}}(k)
\end{aligned}
$$

with

$$
I_{\ell}^{\mathrm{len}}(k)=\int W^{\mathrm{len}}(k, r) j_{l}(k r)
$$

$$
W^{\mathrm{len}}(k, r)=-3 \frac{\Omega_{m}}{a}\left(\frac{H_{0}}{k}\right)^{2} G(r) \frac{d_{A}\left(r_{0}-r\right)}{d_{A}(r) d_{A}\left(r_{0}\right)}
$$

Here I have used the Rayleigh expansion of a plane wave, Eq. (7), and the fact that $\nabla^{2} Y_{l}^{m}=-l(l+1) Y_{l}^{m}$.

For the construction of deflection angles based on the CMB temperature data, I make use of the quadratic statistic proposed by [16] involving the divergence of the temperature weighted temperature gradients, $\nabla \cdot[T(\hat{\mathbf{n}}) \nabla T(\hat{\mathbf{n}})]$. In Fourier space, I can write the estimator for the deflection angles as

$$
\begin{aligned}
D(\mathbf{l})= & \frac{N_{l}}{l} \int \frac{d^{2} \mathbf{l}_{1}}{(2 \pi)^{2}}\left(\mathbf{l} \cdot \mathbf{l}_{1} C_{l_{1}}^{\mathrm{CMB}}+\mathbf{l} \cdot\left(\mathbf{l}-\mathbf{l}_{1}\right) C_{\left|\mathbf{l}-\mathbf{l}_{1}\right|}^{\mathrm{CMB}}\right) \\
& \times \frac{\theta\left(l_{1}\right) \theta\left(\left|\mathbf{l}-\mathbf{l}_{1}\right|\right)}{2 C_{l_{1}}^{\mathrm{tot}} C_{\left|\mathbf{l}-\mathbf{l}_{1}\right|}^{\mathrm{tot}}} .
\end{aligned}
$$

The ensemble average, $\langle D(\mathbf{l})\rangle$, is equal to the deflection angle, $l \phi(\mathbf{l})$, when

$$
N_{l}^{-1}=\frac{1}{l^{2}} \int \frac{d^{2} \mathbf{l}_{1}}{(2 \pi)^{2}} \frac{\left(\mathbf{l} \cdot \mathbf{l}_{1} C_{l_{1}}^{\mathrm{CMB}}+\mathbf{l} \cdot\left(\mathbf{l}-\mathbf{l}_{1}\right) C_{\left|\mathbf{l}-\mathbf{l}_{1}\right|}^{\mathrm{CMB}}\right)^{2}}{2 C_{l_{1}}^{\mathrm{tot}} C_{\left|\mathbf{l}-\mathbf{l}_{1}\right|}^{\mathrm{tot}}} .
$$

Note that $N_{l}$ is the noise power spectrum associated with the reconstructed deflection angle power spectrum:

$$
\left\langle D(\mathbf{l}) D\left(\mathbf{l}^{\prime}\right)\right\rangle=(2 \pi)^{2} \delta_{D}\left(\mathbf{l}+\mathbf{l}^{\prime}\right)\left(l^{2} C_{l}^{\phi \phi}+N_{l}\right) .
$$

In the case of lensing surveys using galaxy shear data, we rewrite Eqs. (37) and (39) such that $d_{A}\left(r_{0}\right)=d_{A}\left(r_{s}\right)$ where $r_{s}$ is the radial distance to background sources from which shape measurements are made. I assume that all sources are at the same redshift, though a distribution of sources in the redshift range expected does not lead to a significantly different result than the one suggested here.

The shot-noise contribution to the convergence power spectrum associated with lensing surveys involving galaxy ellipticity data is

$$
C_{l}^{\mathrm{N}_{\mathrm{X}}}=\frac{\left\langle\gamma_{\mathrm{int}}^{2}\right\rangle}{\bar{n}}
$$

where $\left\langle\gamma_{\text {int }}^{2}\right\rangle^{1 / 2}$ is the rms noise per component introduced by intrinsic ellipticities, typically $\sim 0.6$ for best ground based surveys, and $\bar{n}$ is the surface number density of background source galaxies from which shape measurements can be made. For surveys that reach a limiting magnitude in $R$ $\sim 25$, the surface density is consistent with $\bar{n} \sim 6.9$ $\times 10^{8} \mathrm{sr}^{-1}$ or $\approx 56 \mathrm{gal}$ arc $\min ^{-2}$ [36], such that $C_{l}^{\mathrm{N}} \sim 2.3$ $\times 10^{-10}$.

In Fig. 4 I compare the lensing convergence power spectrum associated with CMB (top curve) and a large scale structure weak lensing survey from galaxy ellipticities with 


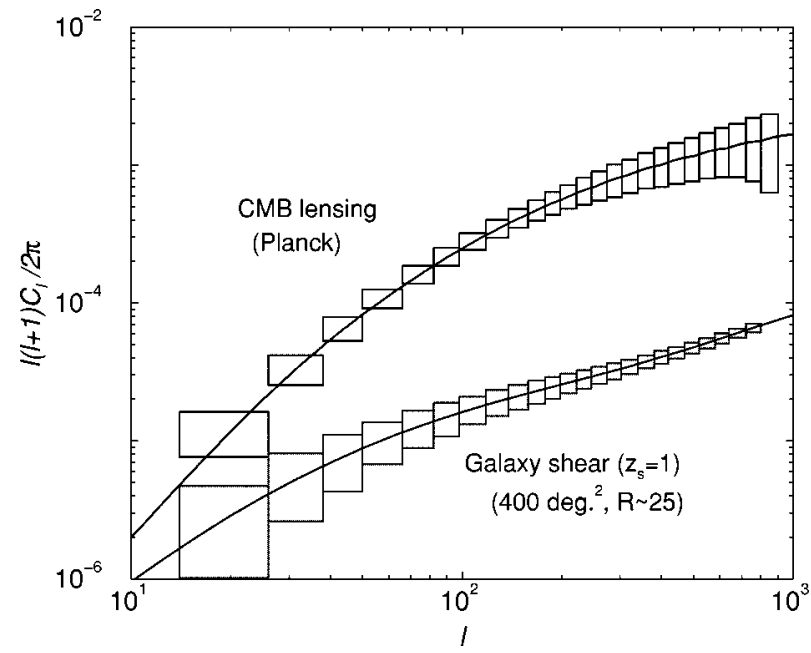

FIG. 4. The power spectrum of convergence constructed from CMB deflections (top curve) and galaxy shape data (bottom curve). In the case of reconstruction based on $\mathrm{CMB}$, I show expected errors from the Planck mission while for large scale structure weak lensing, I show expected errors for a survey of $400 \mathrm{deg}^{2}$ down to an $R$ band magnitude of 25 .

background source galaxies at a redshift of one. Note that I have obtained the convergence power spectrum associated with lensing deflections in CMB following the estimator for the lensing deflection power spectrum and the two are simply related following Eq. (37) such that $C_{l}^{\kappa}=l^{4} / 4 C_{l}^{\phi \phi}$. For comparison, I also show expected error bars on the reconstructed convergence power spectrum from $\mathrm{CMB}$, via the Planck temperature data, and for a wide-field survey of $400 \mathrm{deg}{ }^{2}$ down to an $R$-band magnitude of 25 .

In Fig. 5 I show the associated cumulative signal-to-noise ratio in the detection of the ISW-lensing correlation for galaxy lensing surveys using CMB data. I assume an area of $\pi$ steradians for the lensing surveys and as the signal-to-noise ratio scales as $f_{\text {sky }}^{1 / 2}$, I do not expect a significant use of the current and upcoming lensing surveys which are restricted to at most a few hundred square degrees. The dedicated instruments, such as the Large-aperture Synoptic Survey Telescope (LSST; [37]), however, will provide wide-area maps of the lensing convergence and these will certainly be useful for cross-correlation studies with CMB to extract the ISW effect.

Note that the Planck data allow the best opportunity to detect the ISW effect by correlating an estimator for deflections with a temperature map. The MAP has a lower cumulative signal-to-noise ratio as the estimator for the deflection angle is affected by the low resolution of the temperature data. Nevertheless, the MAP data will certainly allow the first opportunity to detect the presence of the ISW effect either from CMB data alone or through cross-correlation of other tracers.

\section{SUMMARY}

I discussed the correlation between late-time integrated Sachs-Wolfe (ISW) effect in the cosmic microwave background $(\mathrm{CMB})$ temperature anisotropies and the large scale

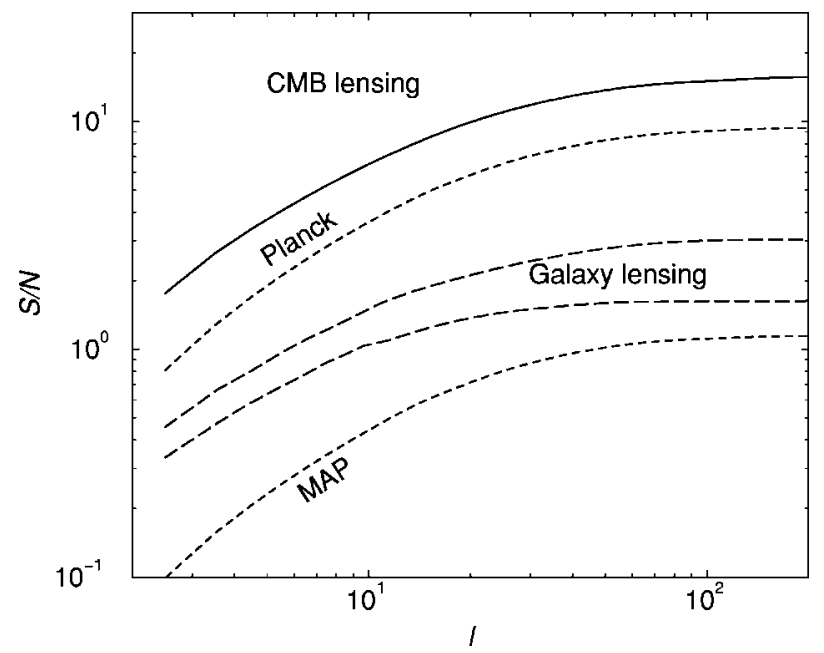

FIG. 5. The signal-to-noise ratio for the detection of lensingISW cross-correlation with galaxy shear data and using deflections in the CMB. The dotted lines are for galaxy weak lensing surveys with background sources at redshifts of 0.5 and 1.5 , respectively, and with a sky area of $\pi$ steradians, similar to the Sloan survey. The dashed lines show the signal-to-noise ratio when temperature data is cross-correlated with an estimator for lensing deflections in Planck and MAP temperature data. The solid line is when a temperature map is cross-correlated with a noise-free estimator of deflections.

structure of the local universe. This correlation has been proposed and studied in the literature as a probe of the dark energy and its physical properties. We considered a variety of large scale structure tracers suitable for a detection of the ISW effect via a cross-correlation.

I summarize our results for the correlation coefficient in Fig. 6. As shown, the potentials that deflect CMB photons

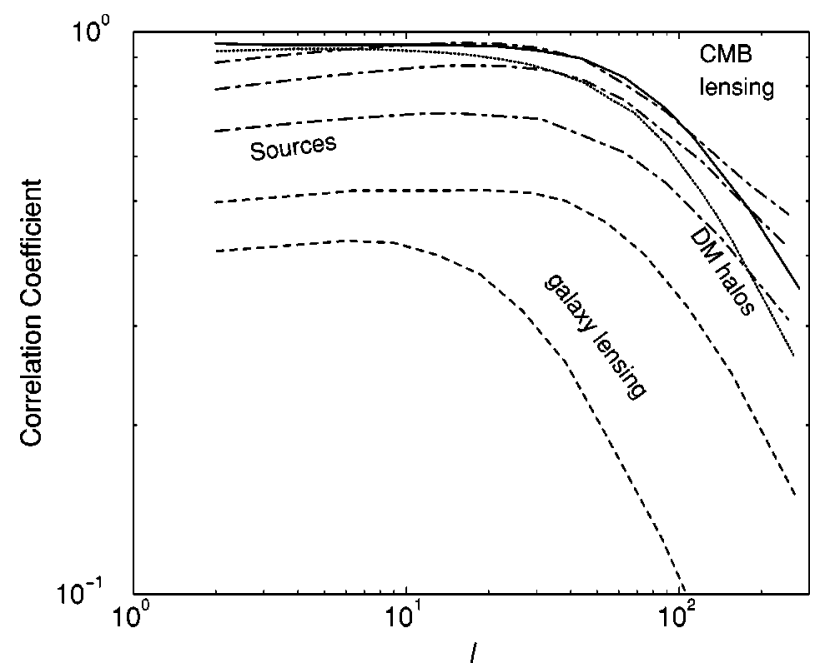

FIG. 6. The correlation-coefficient for ISW-large scale structure correlations involving lensing effect on CMB (solid line), a catalogue of dark matter halos down to a mass limit of $10^{14} \mathrm{M}_{\odot}$ at all redshifts (dotted line), lensing convergence from galaxy shear data (dashed lines) with sources at redshifts of 0.5 and 1.5, and sources as tracers with mean redshifts of $0.4,0.7$, and 1.3. The potentials that deflect $\mathrm{CMB}$ photons and sources at $z \sim 1.5$ are best correlated with the ISW effect. 
are strongly correlated with the ISW effect with correlation coefficients of order 0.95 at multipoles of $\sim 10$. The convergence maps constructed from galaxy shear data are not strongly correlated due to mismatches in their window function and that of the ISW effect. While catalogues of dark matter halos, or galaxy clusters, are well correlated with the ISW effect, sources at low redshifts are necessarily not. Increasing the mean source redshift to $\sim 1.5$, we find correlation coefficients of $\sim 0.9$ suggesting that a catalogue of sources at such high redshifts are preferred over a low redshift survey. Such catalogues can be constructed from surveys at $\mathrm{x}$-ray and radio wavelengths, however, the surface density of such sources are significantly lower than optical galaxies and the resulting cumulative signal-to-noise ratios are subsequently smaller.

In order to understand the correlation between the ISW effect and large scale structure tracers, I rewrite Eq. (10) in the form $\int d k / k k^{3} H(k)$. In Fig. 7 I show $k^{3} H(k)$ as a function of $k$ for large scale structure tracers involving sources and the ISW effect with $l=2$. For comparison, I also show the contribution to potential power spectrum that deflect CMB photons. As shown, there is a mismatch between the wave numbers that contribute to the galaxy clustering, at low redshifts, when compared to the ISW effect. With an increase in source redshift, the mismatch decreases and the sources tend to be more correlated with the ISW effect. On the other hand, it is clear that lensing potentials that deflect CMB photons are well correlated with the ISW effect. In fact, I find that the Planck survey data alone provide the best opportunity to extract the ISW effect using an estimator of lensing deflections on its temperature data. With multifrequency data, the presence of the ISW-large scale structure correlation can also be investigated through a cross-correlation of the frequency cleaned SZ and CMB maps.

In the near term, the ISW-tracer correlation is best studied through catalogues of clusters selected based on mass. Such catalogues can be constructed with upcoming wide-field lensing, SZ effect, and x-ray imaging surveys. The combined

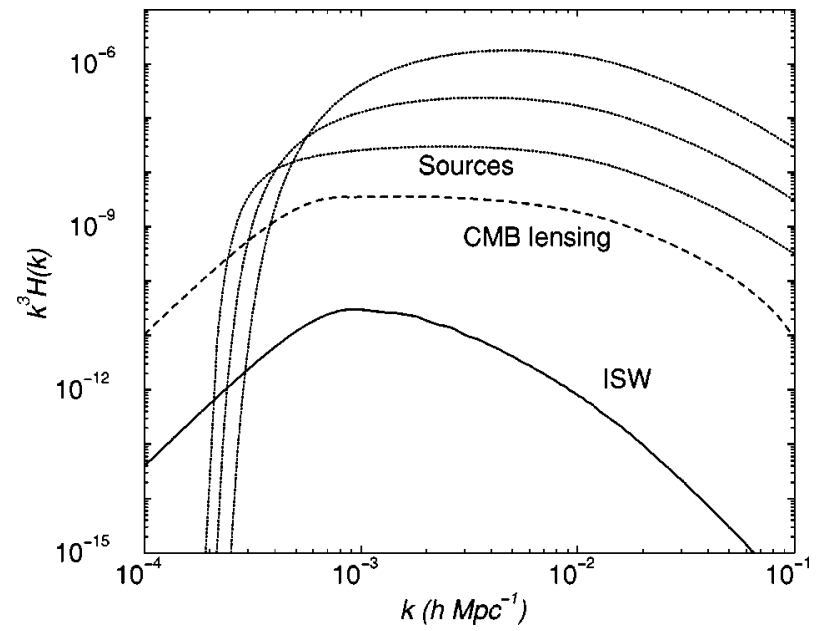

FIG. 7. The contribution to the power spectra of ISW effect and other tracers as indicated. The sources have a mean redshift, $z_{0}$, of $0.4,0.7$, and 1.3 (from top to bottom). The plot shows that the contribution to the lensing effect on CMB comes from the same Fourier modes as the ISW effect while there is a mismatch when compared to galaxy surveys at low redshifts.

DUET cluster catalogue and the MAP data will allow a good opportunity to study the ISW-large scale structure correlation while MAP data can also be used with the Sloan catalogue of galaxies. I estimate the signal-to-noise ratio of order few for these scenarios suggesting that any such detection will be challenging. For DUET, attempts to improve the shot-noise on the X-ray side will be necessary while for the Sloan survey, one should carefully select a subsample of high redshift galaxies, say through photometric redshift data.

\section{ACKNOWLEDGMENTS}

This work was supported at Caltech by the Sherman Fairchild foundation and the DOE grant DE-FG03-92-ER40701.
[1] L. Knox, Phys. Rev. D 52, 4307 (1995); G. Jungman, M. Kamionkowski, A. Kosowsky, and D.N. Spergel, ibid. 54, 1332 (1995); J.R. Bond, G. Efstathiou, and M. Tegmark, Mon. Not. R. Astron. Soc. 291, L33 (1997); M. Zaldarriaga, D.N. Spergel, and U. Seljak, Astrophys. J. 488, 1 (1997); D.J. Eisenstein, W. Hu, and M. Tegmark, ibid. 518, 2 (1999).

[2] W. Hu, N. Sugiyama, and J. Silk, Nature (London) 386, 37 (1997); P.J.E. Peebles and J.T. Yu, Astrophys. J. 162, 815 (1970); R.A. Sunyaev and Ya.B. Zel'dovich, Astrophys. Space Sci. 7, 3 (1970); J. Silk, Astrophys. J. 151, 459 (1968); see a recent review by W. Hu and S. Dodelson, astro-ph/0110414.

[3] R.K. Sachs and A.M. Wolfe, Astrophys. J. 147, 73 (1967).

[4] M.J. Rees and D.N. Sciama, Nature (London) 519, 611 (1968); U. Seljak, Astrophys. J. 460, 549 (1996); also, R. Tuluie and P. Laguna, Astrophys. J. Lett. 445, L73 (1995); R. Tuluie, P. Laguna, and P. Anninos, Astrophys. J. 463, 15 (1996); A. Cooray, Phys. Rev. D 65, 083518 (2002).
[5] A. Blanchard and J. Schneider, Astron. Astrophys. 184, 1 (1987); A. Kashlinsky, Astrophys. J. Lett. 331, L1 (1988); E.V. Linder, Astron. Astrophys. 206, 1999 (1988); S. Cole and G. Efstathiou, Mon. Not. R. Astron. Soc. 239, 195 (1989); M. Sasaki, ibid. 240, $415 \quad$ (1989); K. Watanabe and K. Tomita, Astrophys. J. 370, 481 (1991); M. Fukugita, T. Futumase, M. Kasai, and E.L. Turner, ibid. 393, 3 (1992); L. Cayon, E. Martinez-Gonzalez, and J. Sanz, ibid. 413, 10 (1993); U. Seljak, ibid. 463, 1 (1996); M. Zaldarriaga and U. Seljak, Phys. Rev. D 58, 023003 (1998).

[6] W. Hu, Phys. Rev. D 62, 043007 (2000).

[7] W. Hu and A. Cooray, Phys. Rev. D 63, 023504 (2000).

[8] R.A. Sunyaev and Ya.B. Zel'dovich, Mon. Not. R. Astron. Soc. 190, 413 (1980).

[9] J.P. Ostriker and E.T. Vishniac, Nature (London) 322, 804 (1986); E.T. Vishniac, Astrophys. J. 322, 597 (1987); S. Dodelson and J.M. Jubas, ibid. 439, 503 (1995); G. Efstathiou, in 
Large Scale Motions in the Universe. A Vatican Study Week, edited by V.C. Rubin and G.V. Coyne (Princeton University, Princeton, NJ, 1988), p. 299; A.H. Jaffe and M. Kamionkowski, Phys. Rev. D 58, 043001 (1998); W. Hu, Astrophys. J. 529, 12 (1999).

[10] W. Hu and N. Sugiyama, Phys. Rev. D 50, 627 (1994).

[11] R.G. Crittenden and N. Turok, Phys. Rev. Lett. 76, 575 (1996); A. Kinkhabwala and M. Kamionkowski, ibid. 82, 4172 (1999).

[12] S. Boughn, R. Crittenden, and N. Turok, New Astron. 3, 275 (1998); S. Boughn and R.G. Crittenden, Phys. Rev. Lett. 88, 021302 (2001).

[13] H.V. Peiris and D.N. Spergel, Astrophys. J. 540, 605 (2000).

[14] L. Verde and D.N. Spergel, Phys. Rev. D 65, 043007 (2002); W. Hu, ibid. 65, 023003 (2002).

[15] U. Seljak and M. Zaldarriaga, Phys. Rev. D 60, 043504 (1999); U. Seljak and M. Zaldarriaga, Phys. Rev. Lett. 82, 2636 (1999); M. Zaldarriaga and U. Seljak, Phys. Rev. D 59, 123507 (1999); J. Guzik, U. Seljak, and M. Zaldarriaga, ibid. 62, 043517 (2000); K. Benabed, F. Bernardeau, and L. van Waerbeke, ibid. 63, 043501 (2001); W. Hu, ibid. 64, 083005 (2001); W. Hu and T. Okamato, astro-ph/0111606.

[16] W. Hu, Astrophys. J. Lett. 557, L79 (2001).

[17] J.M. Bardeen, Phys. Rev. D 22, 1882 (1980).

[18] P.J.E. Peebles, The Large-Scale Structure of the Universe (Princeton University, Princeton, NJ, 1980).

[19] D.J. Eisenstein and W. Hu, Astrophys. J. 511, 5 (1999).

[20] D. Limber, Astrophys. J. 119, 655 (1954).

[21] E.F. Bunn and M. White, Astrophys. J. 480, 6 (1997).

[22] P.T.P. Viana and A.R. Liddle, Mon. Not. R. Astron. Soc. 303, 535 (1999).

[23] A.J. Benson, S. Cole, C.S. Frenk, C.M. Baugh, and C.G. Lacey, Mon. Not. R. Astron. Soc. 311, 793 (2000).

[24] L. Verde et al., astro-ph/0112161; H.A. Feldman, J.A. Frieman, J.N. Fry, and R. Scoccimarro, Phys. Rev. Lett. 86, 1434 (2001).

[25] R.J. Scherrer and E. Bertschinger, Astrophys. J. 381, 349
(1991); R.K. Sheth and B. Jain, Mon. Not. R. Astron. Soc. 285, 231 (1997); U. Seljak, ibid. 318, 203 (2000); C.-P. Ma and J.N. Fry, Astrophys. J. 543, 503 (2000); A. Cooray and W. Hu, ibid. 548, 7 (2001); R. Scoccimarro, R. Sheth, L. Hui, and B. Jain, ibid. 546, 20 (2001); J.A. Peacock and R.E. Smith, Mon. Not. R. Astron. Soc. 318, 1144 (2000).

[26] J.J. Condon et al., Astron. J. 115, 1693 (1998).

[27] D.G. York et al., Astron. J. 120, 1579 (2000).

[28] See, for example, G.P. Holder, Z. Haiman, and J. Mohr, Astrophys. J. 553, 545 (2001).

[29] W.H. Press and P. Schechter, Astrophys. J. 187, 425 (1974); R.K. Sheth and G. Tormen, Mon. Not. R. Astron. Soc. 308, 119 (1999).

[30] A. Jenkins, C.S. Frenk, S.D.M. White, J.M. Colberg, S. Cole, A.E. Evrard, H.M.P. Couchman, and N. Yoshida, Mon. Not. R. Astron. Soc. 321, 372 (2001).

[31] H.J. Mo, Y.P. Jing, and S.D.M. White, Mon. Not. R. Astron. Soc. 284, 189 (1997); H.J. Mo and S.D.M. White, ibid. 282, 347 (1996).

[32] J.P. Henry, Astrophys. J. 534, 565 (2000); V.R. Eke, S. Cole, and C.S. Frenk, Mon. Not. R. Astron. Soc. 282, 263 (1996); T.T. Nakamura and Y. Suto, Prog. Theor. Phys. 97, 49 (1997).

[33] A. Cooray, Ph.D. thesis, University of Chicago, 2001; available from the U. of Chicago Crear Science Library or from the author; A. Cooray, Phys. Rev. D 64, 063514 (2001).

[34] A. Cooray, W. Hu, and M. Tegmark, Astrophys. J. 540, 1 (2000).

[35] N. Kaiser, Astrophys. J. 388, 272 (1992); 498, 26 (1998); M. Bartelmann and P. Schneider, Phys. Rep. 340, 291 (2001).

[36] I. Smail, S.W. Hogg, L. Yan, and J.G. Cohen, Astrophys. J. 449, 105 (1995).

[37] A. Tyson and R. Angel, in New Era of Wide-Field Astronomy, edited by R. Clower, A Adamson, and G. Bromage, ASP Conference Series Vol. 232 (Astronomical Society of the Pacific, San Francisco, 2001), p. 347. 